\title{
Brushing as good as handpiece prophylaxis before placing sealants
}

\author{
Abstracted from \\ Kolavic Gray S, Griffin SO, Malvitz DM, Gooch BF.
}

A comparison of the effects of toothbrushing and handpiece prophylaxis on retention of sealants. J Am Dent Assoc 2009; 140: 38-46. Review. Erratum in: / Am Dent Assoc 2009; 140: 1352.

Address for correspondence: Dr Gooch, Surveillance, Investigations, and Research Branch, Division of Oral Health, Centers for Disease Control and Prevention, 4770 Buford Highway, Mailstop F10, Chamblee, Ga. 30341,USA. E-mail: bfg1@cdc.gov

\section{Questions: Is sealant retention as good following tooth surface cleaning with a toothbrush compared with handpiece prophylaxis?}

\section{Data sources Medline and The Cochrane Library.}

Study selection Randomised controlled trials or systematic reviews that considered retention or the effectiveness of resin-based sealants after different surface-cleaning procedures were included. Only English language studies were considered.

Data extraction and synthesis Data were extracted by one author and a summary retention rate calculated separately for the studies that used the same type of surface-cleaning method or toothbrush prophylaxis. The studies were weighted by the reciprocal of their squared standard error.

Results All of the sealant manufacturers' instructions for use (IFU) recommended cleaning the tooth before acid etching. None of the IFU directly stated that a handpiece was required to perform the cleaning, but five IFU implied the use of handpiece prophylaxis. None of the IFU recommended surface-altering procedures in caries-free teeth. Direct evidence from two clinical trials showed no difference in complete sealant retention between surfaces cleaned mechanically with pumice or prophylaxis paste and those cleaned with air-water syringe or dry toothbrushing. Indirect evidence from 10 studies found that weighted summary retention by year after sealant placement in studies that used toothbrush prophylaxis was greater than or equivalent to values for studies that used handpiece prophylaxis.

Conclusions The results indicate that retention of sealants after a supervised toothbrush cleaning by the patient was at least as high as those associated with a traditional handpiece prophylaxis. These findings may translate into lower costs for materials, equipment and personnel.

\section{Commentary}

This review was undertaken to find whether manual toothbrushing is an adequate substitute for traditional dental handpiece prophylaxis prior to sealant placement. The authors specifically address the issue in relation to school-based dental sealant programmes (SBSP) where the use of toothbrushing rather than a handpiece has the potential for significant cost savings. The choice of what, if any, cleaning agents/pastes should be used was also addressed within the text. The conclusion was that sealant retention following surface cleaning with a toothbrush is as least as effective as handpiece prophylaxis.

There are some aspects of the review which are unusual; for example three data sets were used to address the question. The authors commenced by examining five sealant manufacturers' instructions. The sealants selected for this part of the review were unfilled resins as the authors state these are the most commonly used in SBSP. However, including all available sealants would have been more inclusive. Not unsurprisingly this review of manufacturers' instructions did not yield an answer to the question as these tend to be understandably conservative and have not evolved over time.

A literature search yielded two trials which directly compared surface cleaning methods. Handpiece versus a dry toothbrush ${ }^{1}$ or air/ water (3-in-1) syringe assisted by mechanical cleaning with a probe. ${ }^{2}$ Neither study demonstrated any difference in sealant retention.

An analysis of sealant effectiveness and cleaning methods was also undertaken, drawing the data from published systematic reviews of sealant effectiveness, in order to report indirect associations between sealant retention and cleaning method: this yielded 11 trials. Again use of a toothbrush was as effective as a handpiece. This latter aspect of the review also suggests that the advice to avoid fluoridecontaining pastes in many manufacturers' instructions is irrelevant, which is supported by other work. ${ }^{3}$ What does this review tell us, on admittedly limited information? Firstly, the method of cleaning of the surface used does not appear to influence sealant retention rates. Secondly, the use of or type of cleaning agent or paste used does not appear to have an influence.

The authors do outline the limitations of the review: however, one methodological point not addressed is why did only one author reviewed the abstracts for inclusion and one abstracted the data, rather than the normal two? Although given the nature of the data I doubt this has influenced the validity of the findings.

Perhaps the authors of the paper could have widened the question to: is there any need to brush teeth before sealing? As one of the 


\section{CARIES}

two trials identified suggests there is not. ${ }^{2}$ This raises the question; is there an amount of plaque present on a surface which the acid is incapable of burning off and still etching the surface adequately, as is the case for self etching adhesive systems? ${ }^{4}$ Therefore indicating a threshold at which mechanical cleaning is required.

Other questions we need more high quality research to answer include: confirmation of the findings of the review; also if you decide to clean teeth by manual brushing does the child brushing their own teeth or the operator doing this have an influence?

In conclusion I feel the authors have answered the question they addressed and in school based sealant programmes manual toothbrushing is an adequate substitute for professional prophylaxis.
Chris Deery

Department of Oral Health and Development, School of Clinical Dentistry, University of Sheffield,

Sheffield, UK

1. Gillcrist JA, Vaughan MP, Plumlee GN Jr, Wade G. Clinical sealant retention following two different tooth-cleaning techniques. / Public Health Dent 1998; 58: 254-256.

2. Donnan MF, Ball IA. A double-blind clinical trial to determine the importance of pumice prophylaxis on fissure sealant retention. Br Dent J 1988; 165: 283-286. 3. Aboush YE, Tareen A, Elderton RJ. Resin-to-enamel bonds: effect of cleaning the enamel surface with prophylaxis pastes containing fluoride or oil. Br Dent / 1991 ; 171: 207-209.

4. Burbridge L, Nugent Z, Deery C. A randomized controlled trial of the effectiveness of a one-step conditioning agent in sealant placement: 6-month results. Int J Paediatr Dent 2006; 16: 424-430.

Evidence-Based Dentistry (2010) 11, 79-80. doi:10.1038/sj.ebd.6400738 